\title{
APPLYING OPTIMAL CHOICES FOR REAL POWERTRAIN AND LIGHTWEIGHTING TECHNOLOGY OPTIONS TO PASSENGER VEHICLES UNDER UNCERTAINTY
}

\author{
Erik Wilhelm ${ }^{1}$, Johannes Hofer ${ }^{2}$, Lynette Cheah ${ }^{3}$ \\ ${ }^{1,3}$ Singapore University of Technology and Design, Singapore \\ ${ }^{2}$ ETH Zürich (Swiss Federal Institute of Technology in Zurich), Switzerland
}

Submitted 2 January 2015; resubmitted 31 March 2015; accepted 13 May 2015

\begin{abstract}
This paper illustrates how cost-constrained optimization based on a set of real lightweighting and powertrain efficiency options can be used to guide decision-making for automotive manufacturers. The paper provides a method for answering the question posed by Original Equipment Manufacturers (OEMs): 'given a maximum amount additional cost which can be passed on to consumers for fuel-saving technology with uncertain manufacturing cost, to what degree should it be spent on lightweighting versus powertrain efficiency improving technology'. The optimization is formulated as a 0-1 knapsack problem, and dynamic programming is used to find the global optimum technology combination at various levels of maximum up-front technology cost. This paper builds on previous work, which showed that for continuous marginal cost functions under uncertainty, a decision heuristic to either implement lightweighting technology or efficiency technology but not both under cost constraints was preferable. This work extends that result to provide more quantitative strategies for dealing with uncertainty, and finds that, despite uncertainty, optimum lightweighting and efficiency technology selections can be made for the real discrete cases studied. It is found that while the optimum efficiency technology set is highly sensitive to the up-front cost a consumer is willing to pay for future operational savings, lightweighting options are often selected preferentially to efficiency reduction measures. In the same sense, although both technologies are very sensitive to discount rate, lightweighting technologies are less sensitive. Fully hybridized vehicles emerge as a robust option, and, surprisingly, rank highly together with fully electric powertrains.
\end{abstract}

Keywords: cost; fuel consumption; models and algorithms; uncertainty; optimization; lightweighting; powertrain efficiency; strategy development.

\section{Introduction}

Auto manufacturers are facing pressures to improve vehicle fuel efficiency, and can turn to reducing vehicle weight or implementing efficiency technologies like downsizing/turbo-charging or electrification to meet targets. Determining the optimal combination of these technical options under cost constraints is not straightforward, however, and earlier work recommended several strategies based on levels of certainty about future costs (Wilhelm et al. 2012). The original work found that if there was substantial uncertainty regarding the final manufacturing costs for an advanced fuel-saving technology, a decision heuristic to either implement all available efficiency measures, or all available lightweighting measures, but not both, within the cost constraints would be advisable. The previous work also proposed an optimization framework, which could be applied under the assumption that continuous marginal cost functions could be derived from inherently discrete technology choices. This paper examines similar questions, but employs a more realistic discrete choice optimization method, uses new and more credible technology cost data gleaned from real market prices and rigorous analysis, and presents a comprehensive uncertainty analysis. This paper also restricts itself to a specific vehicle platform for each technology measure to ensure that the cost comparisons can be made fairly, and therefore consists of theory and a relevant case study.

Manufacturers are making significant investments in advanced technologies to both meet consumer demand for fuel efficiency (Allcott, Wozny 2012) as well as to meet mandated emissions targets (Klier, Linn 2012). There are several vehicles which have recently been put into limited production, such as the $B M W$ i3 (Rams-

Corresponding author: Erik Wilhelm

E-mails: erikwilhelm@sutd.edu.sg, erik.wilhelm@gmail.com 
brock et al. 2013) and the Volkswagen Up! (Lieberman 2009), which demonstrate manufacturer's willingness to implement a high degree of powertrain efficiency while simultaneously invest in technology to reduce vehicle weight dramatically. This investment comes at a substantial cost which must be passed on to the consumer, who may not be willing to bear it, given what is understood about the willingness to pay for future fuel savings in the automotive market (Greene 2010a). There are of course some cost savings by reducing weight, for example by maintaining range with reduced battery capacity, but for the vehicles, being currently brought to market it is unclear whether these savings are significant.

This paper presents rigorous methods of addressing the question 'given a fixed up-front amount investment in powertrain efficiency technology and/or vehicle lightweighting technology under manufacturing cost uncertainty, which should be chosen?' from a manufacturer's perspective. The implicit assumption made is that other aspects of vehicle purchase decisions such as safety, comfort, prestige, luggage capacity, etc. are able to be maintained regardless of the powertrain or lightweighting technology choices made. The methods presented here are generally applicable to assisting Original Equipment Manufacturer (OEM) decision-making under uncertainty.

\section{Technology Measures}

Whereas the previous work focussed on building functions to describe marginal technology costs, this paper examines two vehicle platforms (Ford Focus and Ford Fusion) as case studies, representing all of the discrete technology options relevant for analysing the optimal lightweighting and efficiency technology mix. The efficiency measures are additionally assumed to be mutually exclusive (i.e. only one can be selected), whereas the lightweighting measures are assumed to be able to be implemented without any exclusions.

\subsection{Lightweighting}

The 19 lightweighting technology options will be based on a comprehensive 2012 Environmental Protection Agency (EPA) study detailing the tear-down of a 2010 midsize crossover vehicle (FEV 2012). Together with the null option, the optimization routine can select between a total of 20 weight reduction options. These include reducing the weight of different subsystems like the engine, transmission and exhaust systems. The reported marginal cost of weight reduction (in \$ per kg reduced) and the effective vehicle weight reduction is visualized in Fig. 1.

Appendix A tabulates all of the options, which can be considered by the optimization routine, and provides additional data about each of the technology options.

It should be noted that no consideration of inertial weight effects during dynamic operation is made in this study.

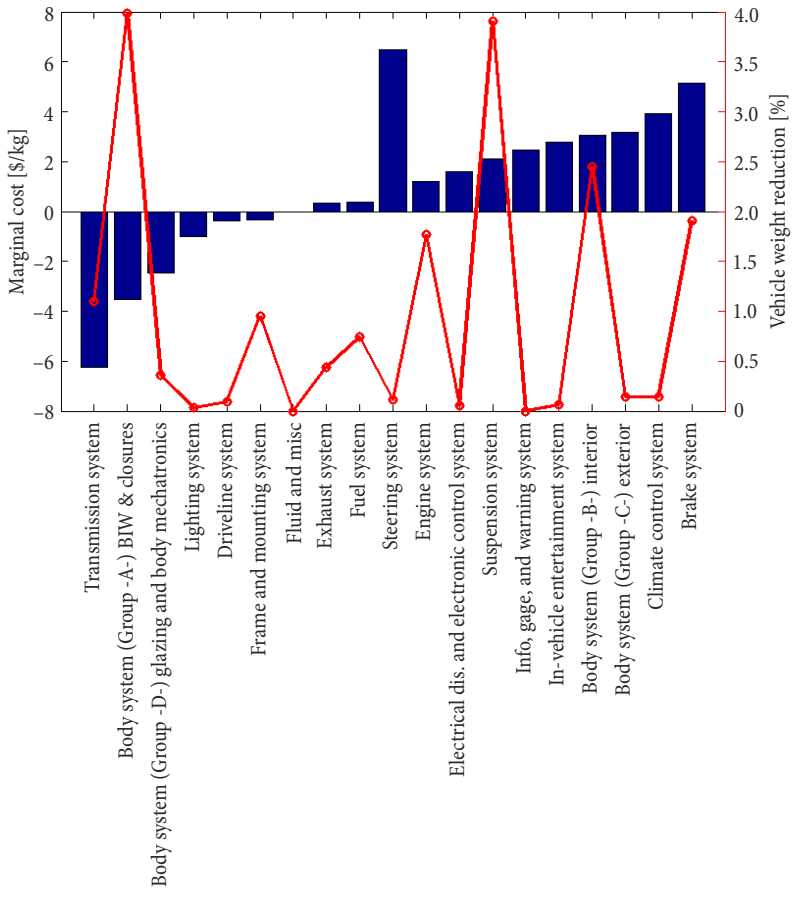

Fig. 1. Marginal costs and associated weight reduction considered (FEV 2012)

\subsection{Efficiency}

The efficiency cost estimation outlined in this section draws on data from 24 vehicles from the same market segment and 2014 model year, and examines fuel efficiency technology applied. For this work, Ford model offerings in the US were selected to compare the costs of various efficiency measures on a common platform from a common manufacturer. All of the efficiency technologies outlined in Table 1 are applied to 2014 Ford Fusion variants. A constant margin factor of 1.4 was used to deduce the manufacturing costs from Manufacturer's Suggested Retail Price (MSRP), the retail price. The authors acknowledge the reality that this ignores important price variations which manufacturers apply when marketing hybridization options, and also biases efficiency technology choices. It is hoped that the methods here can be applied to study baseline vehicles from other manufactures. The uncertainty in marginal cost will be examined in a later section, and we encourage future investigations to consider using more detailed approaches to estimate the marginal costs of efficiency technologies should more valuable data become available.

Appendix B contains all of the options which can be considered. The Ford Focus electric was selected as the Ford Fusion model is not available with an all-electric powertrain. The most substantial cost uncertainty exists for the electric drive vehicle, as it is highly likely that manufacturers are selling these vehicles with dramatically reduced margins or at losses to gain market share for this early-stage technology.

It should also be noted that the data derived from EPA fuel consumption testing shown in Table 1 and used later in this work underestimate fuel consumption, 
which means that the analysis presented in this paper regarding lifetime fuel costs are on the conservative side. Real-world use will result in greater savings and hence greater efficiency improvements should be preferred. For simplicity, no correction factors were used to adjust test-cycle fuel consumption results.

Table 1. Marginal costs of efficiency technology based on MSRP with a constant $40 \%$ mark-up

\begin{tabular}{|l|c|c|}
\hline \multicolumn{1}{|c|}{$\begin{array}{c}\text { Efficiency } \\
\text { technology }\end{array}$} & $\begin{array}{c}\text { Efficiency improvement } \\
\text { relative to baseline } \\
{[\mathrm{L} / 100 \mathrm{~km}]}\end{array}$ & $\begin{array}{c}\text { Marginal } \\
\text { cost [\$] }\end{array}$ \\
\hline Baseline (None) & 0.0 & 10.71 \\
\hline Start-stop (S-S) & 0.3 & 567.86 \\
\hline $\begin{array}{l}\text { Downsize/turbo } \\
\text { (DS/T) }\end{array}$ & 0.6 & 2389.29 \\
\hline Full hybrid (Full) & 4.0 & 6417.86 \\
\hline $\begin{array}{l}\text { Plug-in hybrid } \\
\text { (Hybrid) }\end{array}$ & 5.4 & 8025.00 \\
\hline Electric drive (EV) & 6.8 & \\
\hline
\end{tabular}

\section{Mathematical Formulation}

The sensitivity to powertrain efficiency is driven by Eq. (1) which was developed by Guzzella and Sciarretta (2015) and used extensively in the previous work by Wilhelm et al. to explore general trends in weight and efficiency trade-offs:

$$
\Delta E=\frac{1}{\eta} \cdot(A+(B+C) \cdot \Delta m),
$$

where: $\Delta E$ is the change in fuel consumption for a vehicle caused by the change in mass $\Delta m$ over a specific driving cycle parameterized by $A, B$, and $C$ with average efficiency $\eta$. Note that coefficients used in Eq. (1) neglect regeneration effects present in electric drivetrains. Regeneration would serve to further diminish the impact of mass changes on highly efficient powertrains, but are not considered here.

This formula makes clear that a change in mass has an effect on the change in energy consumption, which diminishes with increasing average powertrain efficiency, as shown in Fig. 2 for a $100-\mathrm{kg}$ mass increase. In essence, the sensitivity of a vehicle to a fixed $100-\mathrm{kg}$ increase in mass diminishes with increased powertrain efficiency, all other vehicle characteristics held equal.

The observation that more efficient vehicles are less sensitive to changes in vehicle mass has been made in several previous works. Cheah et al. (2009) found a relationship of $0.3 \mathrm{~L} / 100 \mathrm{~km}$ for $100-\mathrm{kg}$ weight reduction through powertrain simulation. Wilhelm and Schenler (2010) found a sensitivity of $0.6 \mathrm{~L} / 100 \mathrm{~km}$ for conventional vehicles, a $0.4 \mathrm{~L} / 100 \mathrm{~km}$ for hybrids, and $0.2 \mathrm{~L} / 100 \mathrm{~km}$ for electric vehicles to a $100-\mathrm{kg}$ weight reduction from real-world fuel efficiency data, consistent with the fact that simulation often underestimates real-world consumption. Neither study considered the constant term ( $y$-intercept of energy use versus mass),

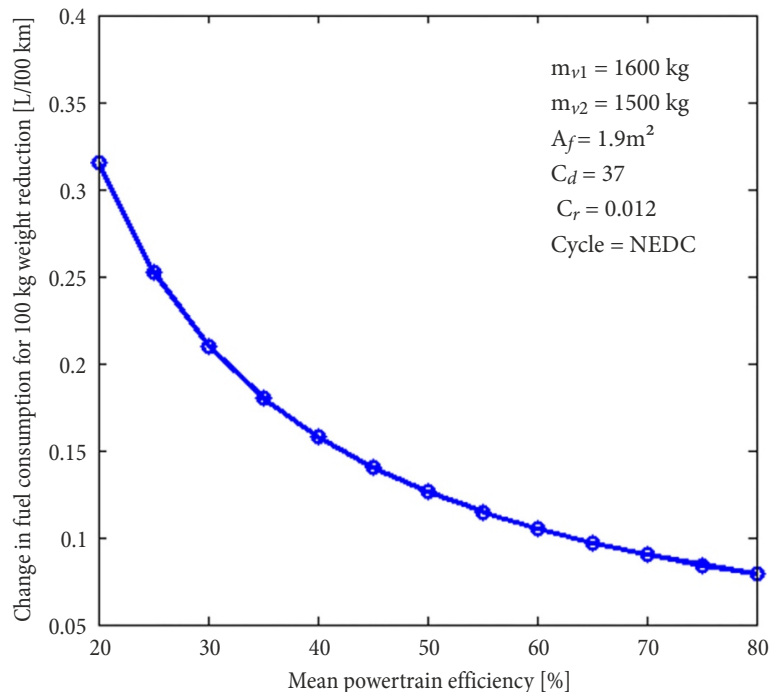

Fig. 2. The sensitivity of a vehicle with various powertrain efficiencies to a $100-\mathrm{kg}$ increase in mass

which is attributed to the drag coefficients as described by Magee (1982), although this oversight does not impact the fundamental argument concerning the impact of efficiency on sensitivity to mass reduction.

To simply illustrate this point, assuming that the vehicle specific energy consumption is described by Eq. (1) it is trivial to show that the ratio of energy change for two powertrains of different average efficiencies is represented by the ratio in Eq. (2) for a change in mass of the same magnitude:

$$
\Delta E_{1}=\frac{\eta_{2}}{\eta_{1}} \cdot \Delta E_{2} .
$$

It is this result, which leads to many of the findings outlined in this paper.

\subsection{Continuous Case}

In the previous paper by Wilhelm et al. (2012) the assumption was made, that a marginal cost function could be parameterized based on the set of discrete technology choices for which data was available. The authors then proceeded to solve the case where marginal costs are static in an analytical way by employing a Heaviside step function. A series of figures with a characteristic 'cliff' were then shown illustrating the effect of this analytical result on the optimal technology mix.

Once marginal cost functions were assumed, the optimization necessitated numerical solution, and several cases were studied which demonstrated equivalent properties to the analytical solution. In that paper, the authors also presented preliminary results of using a simple (and non-globally optimal) discrete solver to illustrate that similar results were obtained. They acknowledged, however, that this was a significant shortcoming of the optimization approach taken, and this realization precipitated the development of the current work. 


\subsection{Discrete Case}

This section presents the method of optimally selecting between discrete lightweighting and efficiency technologies under cost constraints. The problem is formulated to allow the use of a globally optimal 0-1 knapsack algorithm with dynamic programming.

The key assumptions made in this work are:

- there are no mass decompounding effects considered due to vehicle lightweighting being used to revise powertrain sizing (Verbrugge et al. 2009);

- all technology improvement goes towards reducing fuel consumption, i.e. performance, volume, comfort, range etc. are held at constant levels;

- the sensitivity of efficiency to lightweighting is derived from real vehicles (and is embodied in the coefficients $b$ and $k$ described in a later section);

- that gasoline and electricity cost similar amounts on an energy basis; this depends strongly on the grid mix, but the energy-equivalent cost comparison can be made assuming $\$ 0.12 / \mathrm{kWh}$ and $\$ 0.95 / \mathrm{L}$, which are representative US prices (EIA 2012, 2014);

- these values for vehicle operation, which will be further explored in the sensitivity analysis section:

- an 11 year service life,

- $23935 \mathrm{~km}$ driven annually,

- a fuel price of $0.95 \$ / L$

- discount rate of 5\%, calculated according to Net Present Value (NPV) in 2014;

- that Total Cost of Ownership (TCO) is simplified, and only consists of the sum of life-time fuel costs and up-front purchase costs, and ignores maintenance, resale value etc., but will still be referred to as the total cost.

The method used to solve the discrete optimization problem is based on the 0-1 knapsack problem formulation (Martello, Toth 1990), and begins by defining the consumer's TCO according to Eq. (3):

$$
\Delta T C O_{x}=P P+\left(F C_{\text {base }}-\Delta F C_{x}\right) \cdot \frac{F P \cdot k m \cdot \text { life }}{100}+M C_{x} \text {, }
$$

where: $P P$ is the purchase price of the baseline vehicle; $F C$ is the fuel consumption [ $\left.\mathrm{L}_{\text {equivalent }} / 100 \mathrm{~km}\right] ; F P$ is the fuel price $[\$ / \mathrm{L}] ; \mathrm{km}$ is the number of kilometres driven annually; life is the number of years a vehicle is owned for; $M C_{x}$ is the marginal cost of a technology option [ $\$$ ] including a mark-up of $40 \%$.

The optimization approach used in this paper is as follows. First, the baseline vehicle cost $\mathrm{TCO}_{\text {base }}$ with no technology improvement is calculated by setting $M C_{x}$ and $\Delta F C_{x}$ equal to zero to signify that no reduction in efficiency has taken place and thereby no additional costs were incurred.

Next, two matrices, $\Delta F C \in \mathrm{R}^{(2 \times n)}$ and $\Delta m \in \mathrm{R}^{(2 \times m)}$ are defined which contain in their first column associated marginal costs, and the second column of the $n$ efficiency improvements $[\mathrm{L} / 100 \mathrm{~km}]$ and $m[\mathrm{~kg}]$ weight reduction technologies respectively. Rounding each of the up-front costs in the first column according up toward positive infinity results in values stored in new vectors, $\Delta E_{r}$ and $\Delta m_{r}$. Next, all of the technologies in either vector with weight values which are less than or equal to zero are removed, and stored. This is to ensure a positive integer weight is assigned to the marginal costs to be assigned as a weight $w_{k}$ as is required by the knapsack algorithm.

For each $j=1 \ldots n$ in $\Delta E_{r}$ and for each $i=1 \ldots m$ of $\Delta m_{r}$ in a nested loop, use Eq. (4) to determine the reduction in fuel consumption from mass reduction, where the variables $b$ and $k$ represent the intercept and slope of a line generated by plotting real fuel consumption sensitivities $\mathrm{L} / 100 \mathrm{~km} / \mathrm{kg}$ against mean vehicle efficiencies for the data plotted in Fig. 1 of Wilhelm et al. (2012). This dependency is one of the main novelties of the paper, and yields many of the most interesting results. Use Eq. (5) to determine the reduction in fuel consumption from efficiency measures:

$$
\begin{aligned}
& \Delta F C_{i}=\left(b+\left(F C_{\text {base }}+\Delta E_{r, j}\right) \cdot k\right) \cdot \Delta m_{r, i} ; \\
& \Delta F C_{j}=\Delta E_{r, j} .
\end{aligned}
$$

Once all of the fuel consumption reductions for the options are computed according to Eqs (4) and (5), the TCO for each of these options is calculated using Eq. (3). Next, the objective value $v_{k}$ for each $k=1 \ldots n \cdot m$ options are calculated according to Eq. (6), which may take a positive or negative value:

$$
v_{k}=T C O_{b a s e}-\triangle T C O_{k} \text {. }
$$

Once all of the values are calculated, a dynamicprogramming approach based on the 0-1 knapsack problem formulation is applied (Petter 2009). The value $V$ is maximized according to Eq. (7), with the weight constraints according to Eq. (8), where $U$ is the total upfront cost of the technologies selected. The weights $w_{k}$ for the optimization are simply the marginal costs $M C_{x}$ for each technology option rounded to positive infinity from the vectors $\Delta E_{r}$ and $\Delta m_{r}$ :

$$
\begin{aligned}
& \text { Maximize } V=\sum_{k=1}^{n \cdot m} v_{k} \cdot x_{k} ; \\
& \text { subject to } U=\sum_{k=1}^{n \cdot m} w_{k} \cdot x_{k} \leq c_{\max } \\
& \text { with } x_{k}=\left\{\begin{array}{l}
1 \text { if } k \text { selected; } \\
0 \text { if } k \text { not selected; }
\end{array}\right. \\
& k \in N=\{1 \ldots n \cdot m\},
\end{aligned}
$$

where: $c_{\max }$ is the maximum allowable up-front cost. Once the optimization algorithm has been completed, and an optimal set of technologies selected will be denoted $\psi$, the options with weight less than zero are readded to the optimal set, denoted $\psi^{\prime}$. One final check is performed to ensure that the optimization result is valid. Occasionally the efficiency measure in the outer loop for which an efficiency advantage was enjoyed in Eq. (4) was 
not selected. In these cases, the result is deemed invalid, and removed from the set of admissible optimization results. This final set is the optimal set, denoted $\psi^{*}$.

In the final step, the maximum value $V^{*}$ of all the $\mathrm{k}$ efficiency and lightweighting options is selected for the maximum up-front value scenario in question. The total cost for the optimal vehicle technology set $\psi^{*}$ is then determined according to Eq. (9):

$$
\mathrm{TCO}_{\psi^{*}}=\mathrm{TCO}_{\text {base }}-\mathrm{V}^{*} \text {. }
$$

Source code for this algorithm is available from Internet: https://github.com/SUTDMEC/LWvsEff

\section{Results and Discussion}

The brute force approach results in 6291456 designs (6 efficiency technologies $\times 2^{20}$ combinations of lightweighting technologies), and finds that the un-constrained optimum has the lowest cost of $\$ 35094$ found for a vehicle design with full hybridization (split-series parallel but without the plug-in option) and all the lightweight measures applied except for the 'Fluid and misc' measures, which have zero cost and no associated weight reduction. The brute force approach required roughly 12 minutes on a quad-core $2.6 \mathrm{GHz}$ 64-bit Windows machine. By comparison, the 0-1 knapsack problem executes an optimization in only about 0.7 seconds by using a dynamic programming based approach.

The results plotted in Fig. 3 for the brute-force solution to the optimization problem highlight that the search space is discontinuous, and that depending on your willingness to spend up-front a large range of total costs of ownership are possible once operation costs are considered. Note that the implementation of efficiency technology dominates the marginal increase in purchase cost based on the technology set assumed here, but that as the powertrain efficiency increases, the variation in the TCO with various lightweighting technology implementation decreases, once again illustrating how less efficient vehicles are more sensitive to vehicle mass increase (Pagerit et al. 2006; Wohlecker et al. 2007).

The main result of this paper is shown in Fig. 4 where the optimal selection of technologies calculated using the 1-0 knapsack optimization algorithm outlined in the previous section are shown. The two most interesting observations are that (a) for discrete capital investment problems, it is often possible to under-spend as can be expected from the problem formulation and (b) slightly lower TCO may result as some technologies with higher up-front costs are displaced by technology portfolios with lower total up-front costs. For example, if the maximum up-front cost threshold passes beyond $\$ 150$, despite the very large reduction in weight the brake system can contribute, it alone already exceeds the maximum cost threshold and hence will not be selected. In this way, technology selection occurs with increasing preference for high marginal cost reduction. Table 2 shows how the optimization algorithm gradually deselects technology as the maximum allowable up-front

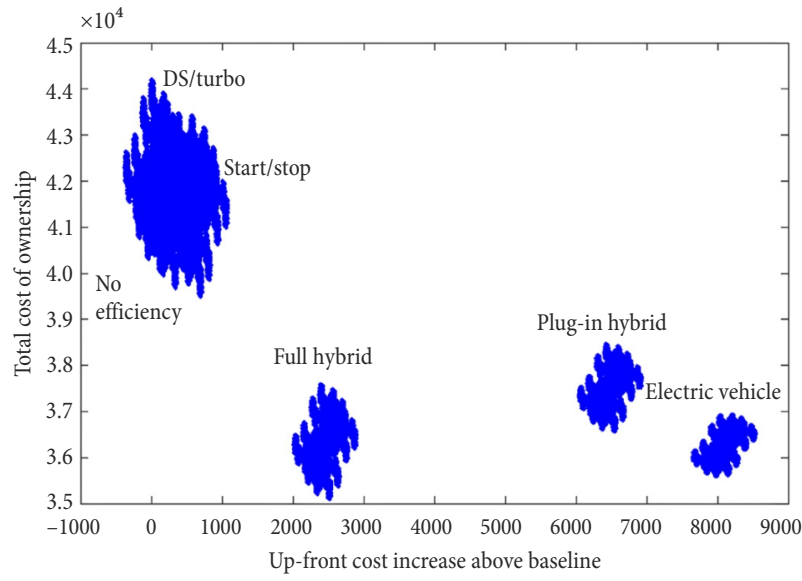

Fig. 3. Brute force approach to the problem finds the TCO for very large set of combinations of efficiency and lightweighting technology ( $0 \%$ discount rate)

a)

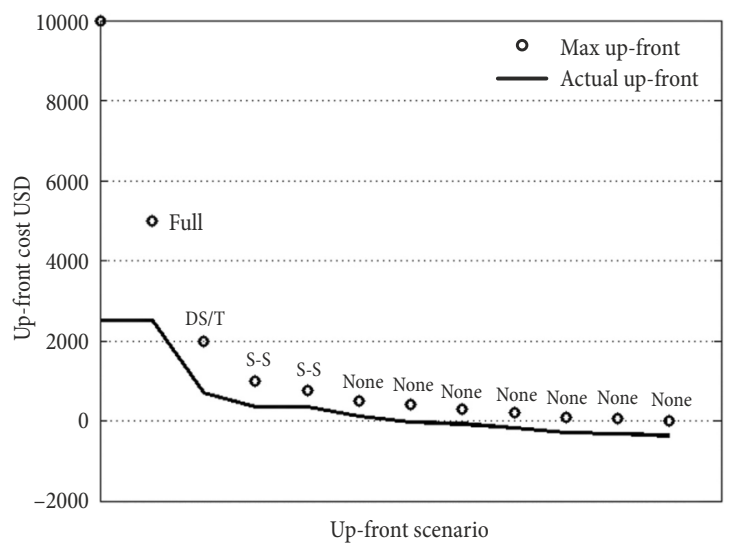

b)

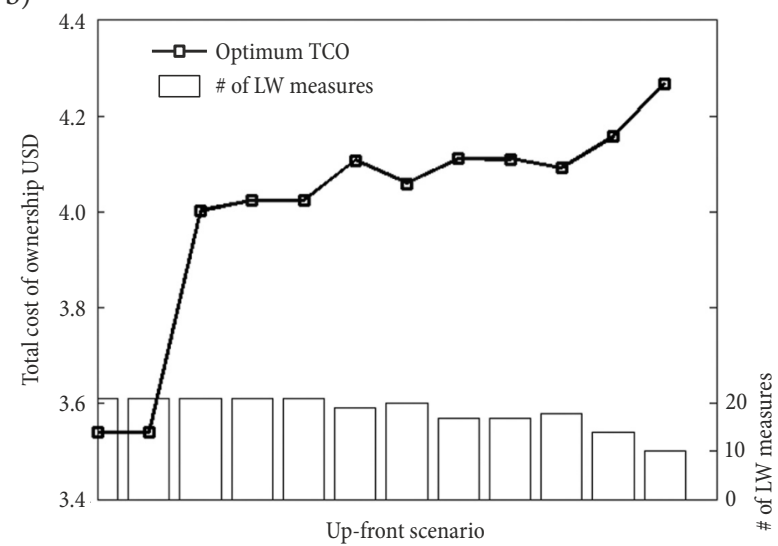

Fig. 4. The 0-1 knapsack dynamic programming optimization algorithm always under-spends, but shows that in several instances up-front cost constraints limit overall performance

cost decreases. Finally, at a maximum allowable cost of $\$ 1$ (the lowest allowed by the optimizer) only the info, gauge and warning system technology, with an integer cost of $\$ 1$, as well as all of the cost-reducing technologies remain selected. 
Table 2. Effect of reducing maximum up-front cost on technology selection

\begin{tabular}{|c|c|}
\hline Max cost $[\$]$ & De-selected lightweighting technology \\
\hline $10000-750$ & None (all LW tech. selected) \\
\hline 500 & Climate Control (CC) and steering \\
\hline 400 & Brake \\
\hline 300 & $\mathrm{CC}$, steering, engine, brake \\
\hline 200 & CC, steering, Group B, brake \\
\hline 100 & Group B, suspension, brake \\
\hline 50 & $\begin{array}{l}\text { CC, steering, Group B, Group C, } \\
\text { suspension, brake, info, gauge and warn }\end{array}$ \\
\hline 1 & $\begin{array}{l}\text { Only LW tech with negative cost, and the } \\
\text { info, gauge and warning system remain } \\
\text { selected }\end{array}$ \\
\hline
\end{tabular}

Unless otherwise stated, the discount rate for all of the analysis performed so far was assumed to be zero, i.e. consumers valued a dollar up-front with the same utility as a future dollar. This is a tenuous assumption, with the research showing a wide variety of consumer behaviour and discount rates, which can be observed (Greene 2010b). The sensitivity of the results to the assumed discount rate is discussed in Section 3.1.

\subsection{Uncertainty Analysis}

\subsubsection{Uncertainty in Cost of Light weighting Technology}

In this section, it is shown that as uncertainty increases, measured by an increase in the standard deviation of a normal vector (Fig. 5), fewer lightweighting technologies are selected. Selecting a maximum technology cost of $\$ 10000$ to allow all efficiency technologies to be selected, full hybrids and EV's are selected with fixed nonstochastic (i.e. completely certain) hybridization costs. A clear trend towards lower numbers of lightweighting technologies with increasing uncertainty can be seen in Fig. 6. This trend persists even if maximum up-front costs are capped at $\$ 7000$ to ensure that only full hybrids are selected. As well, the number of lightweight-

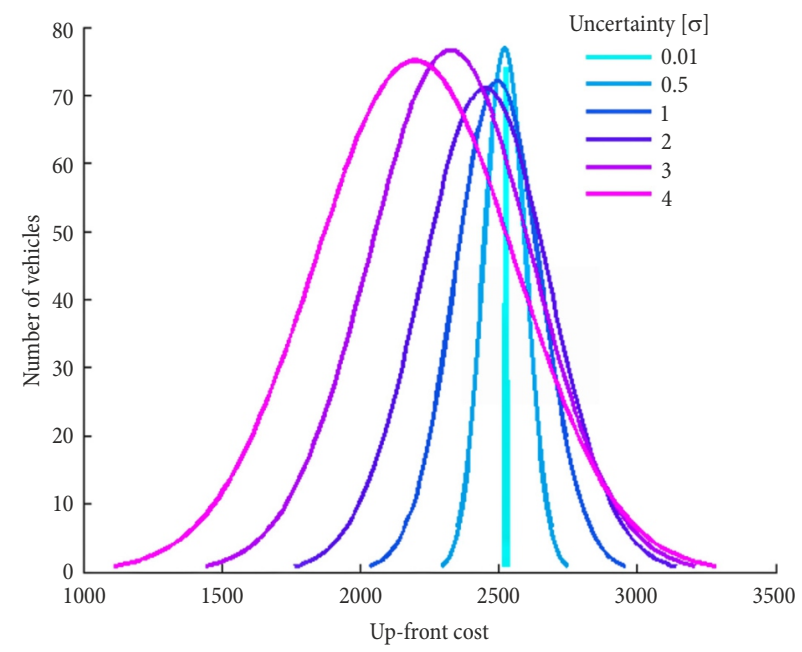

Fig. 5. Up-front cost under increasing uncertainty as a normal distribution ing technologies selected also decreases with increasing uncertainty in the case that lightweighting technology cost is offset to only have positive costs (i.e. removing the potential for cost uncertainty to drive an increase in negative marginal costs).

We assume that the underlying distributions driving manufacturing uncertainty can be arbitrarily defined in this case. Uniform, normal, and triangle distributions were evaluated, and very similar results were obtained with each. Ultimately, a 1000-point Monte Carlo simulation was used for the following analysis, distributed according to the triangle distribution generated using the probability density function given by Eq. (10), as shown in Fig. 7. The stochastic 'cost multiplier' is then used to scale the lightweighting and efficiency cost through simple multiplication:

$$
\text { Cost mult }(x \mid a, b, c)= \begin{cases}\frac{2 \cdot(x-a)}{(c-a) \cdot(b-a)}, & a \leq x \leq b ; \\ \frac{2 \cdot(c-x)}{(c-a) \cdot(c-b)}, & b<x \leq c ; \\ 0, & x<a, x>c,\end{cases}
$$

where: $b=1, a=b-\sigma$ if $b-\sigma$ is greater than 0 , otherwise $a=0$. And $c=b+\sigma$, where $\sigma$ is the uncertainty standard deviation factor being considered (MathWorks 2014).

As the price uncertainty increases for a technology, there will be a greater tendency for the technology to be too expensive to be selected in the knapsack optimisation. It is interesting to note that all of the probability distributions tested in this work are skewed to favour reductions in cost, since any deviations from the mean of 1 , which fall negative are stacked on the positive real value axis which leads to the uncertainty distribution with a skewed tail. Even given this assumption, as uncertainty increases, the average number of lightweighting options selected diminishes.

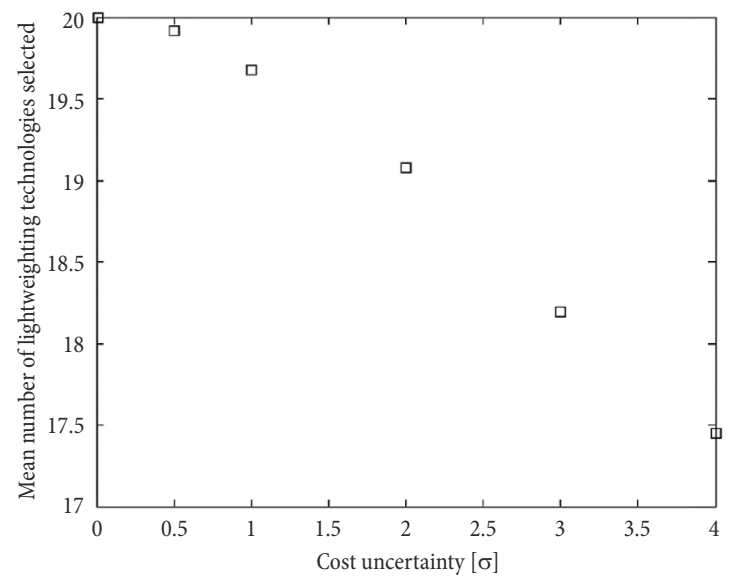

Fig. 6. Increasing stochastic uncertainty in lightweighting cost results in a decreasing number of designs selected for lightweighting 
The trend shown in Fig. 6 and can be explained by understanding that, as uncertainty about a technology's tooling and production cost increases, there is a possibility that its overall NPV becomes negative. This trend is validated by Fig. 8, which shows the effect of uncertainty on the mean number of technologies with negative values as uncertainty increases (over a Monte Carlo of 1000-rounds). Note that the negative NPV is not to be confused with the negative marginal cost. Negative NPV means that the technology no longer contributes to the reduction of the TCO, but rather increases its total cost and is therefore not likely to be in the optimal selection set.

In order to understand these results, various stress tests of the data were performed. It was established that the following effects had no bearing on the trend as the uncertainty increases:

- more technologies with up-front costs which fall below 0 or rise above 0 ;

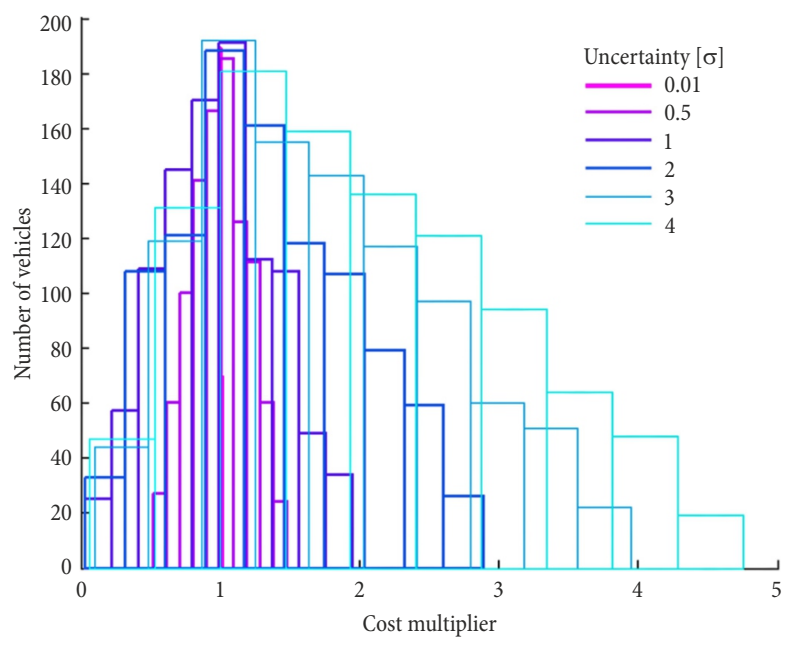

Fig. 7. Input cost multiplier-representing uncertainty according to a triangle distribution, selected for its repeatability and for having a quantifiable moment on the real positive axis

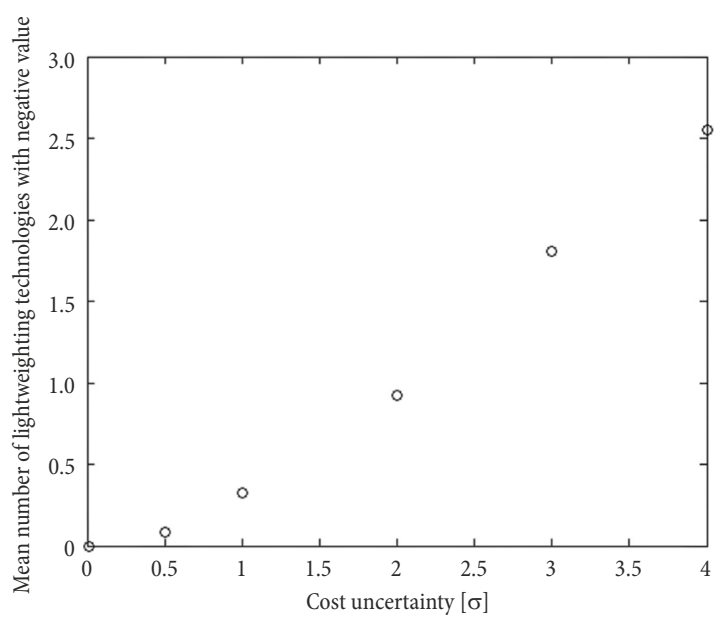

Fig. 8. As uncertainty about marginal up-front costs increase, the mean number of lightweighting technologies with negative values selected increases
- a higher mean value/cost ratio, signifying potentially higher returns for selecting a technology; in fact, the vehicle with the lowest number of lightweighting options selected has a negative mean value/cost ratio;

- that the maximum cost threshold is being exceeded for the selected efficiency measure; in fact the selected measure never exceeds the maximum up-front cost.

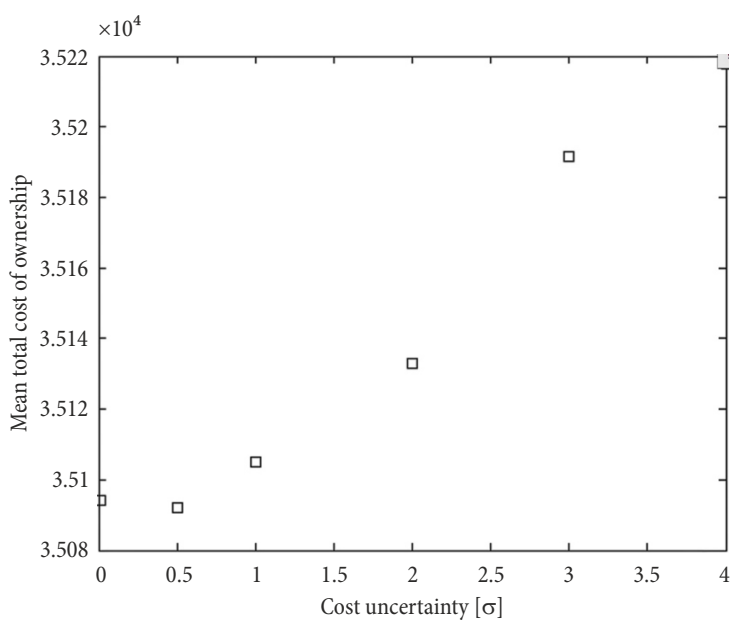

Fig. 9. With increasing up-front cost uncertainty, TCO increases driven by more technologies with negative NPV

The trend in Fig. 9 shows that as up-front cost uncertainty increases, so does the overall TCO. This is consistent with the finding that the probability of negative NPVs increases as uncertainty increases, raising the likelihood that a technology which otherwise may have reduced the TCO no longer does so, and is therefore no longer economical for selection.

\subsubsection{Uncertainty in Cost of Efficiency Technology}

An identical triangle distribution centred on one with increasing variance range identical to the lightweighting technology case described in Fig. 7 was used to generate the multiplier applied to the efficiency technology to simulate cost uncertainty. The cost constraint of $\$ 7000$ was maintained for the following analysis.

Uncertainty in the cost of efficiency technology has the expected effect of obfuscating the optimal powertrain choice, as can be clearly seen in Fig. 10. As uncertainty about marginal efficiency measure cost increases, the variety of optimal powertrain technology increases in the 1000-round Monte Carlo simulation. It was interesting to note the number of EVs, which were selected, indicating that if uncertainty tends to result in lower than expected costs (something which is the triangular distribution has a predilection for), then the EV quickly becomes a competitive option, even when the cap of the maximum upfront cost is 7000, over 1000 less than the base price EV. The mean TCO increased as the uncertainty increases, in a trend, which parallels the lightweighting case. 
The number of lightweighting technologies optimally selected is also affected by the increasing hybridization cost uncertainty, as shown in Fig. 11. This is expected, as the ballooning cost of efficiency measures makes less room for lightweighting to be included in several cases. It is also interesting to see that, even when electric vehicles are selected, the majority of efficiency measures are optimal for selection (although less are selected for the EV case than for the other efficiency measures). This runs slightly counter to the results of the previous paper, which suggested that under uncertain conditions, efficiency measures should be selected in an 'all-or-nothing' fashion. This discrepancy can be explained by the fact that the finding in the previous work was based on a discontinuity resulting from a continuous cost function. This work builds on that observation and performs a more rigorous treatment of the uncertainty associated with the decision-making to determine global optimal choices.

Additionally, the optimal number of lightweighting technologies for more efficient powertrains is lower, as is expected.

\subsection{Sensitivity Analysis}

This section will explore the sensitivity of the models to key assumptions about how many kilometres are driven yearly, what fuel costs, and how many years a vehicle is owned and operated. The findings in this section reflect that the optimal technology choices depend in very similar ways to uncertainty in the input assumptions. For stochastic Monte Carlo simulations, in all three instances, as the impact of yearly cost increases (with increasing driven $\mathrm{km}$, fuel cost, and length of ownership), TCO rises linearly, as is expected through the constitutive relation between these parameters outlined in Eq. (3). For the case where the maximum up-front cost is constrained at $\$ 10000$, the magnitude of the increase always experiences a 'knee point' where the optimal efficiency technology shifts from full hybridization to electric drive. The other condition of the simulation

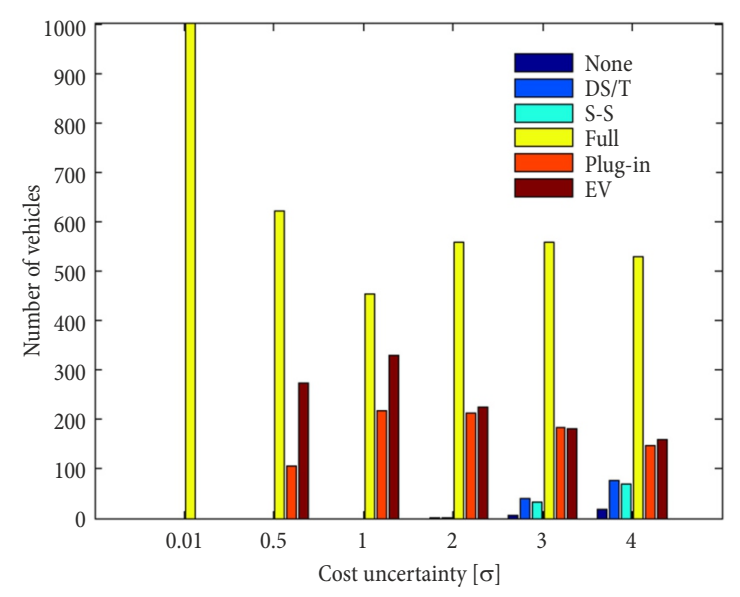

Fig. 10. A wider variety of powertrain technology becomes optimal with increasing marginal cost uncertainty constrains maximum up-front costs to $\$ 1000$. As expected, the sensitivity to further increases in yearly costs is therefore reduced. A conclusion, which can be drawn is that, in general, paying a higher up-front cost is always advantageous relative to lower up-front costs, even with an assumed $0 \%$ discount rate. With a $5 \%$ discount rate, start-stop hybrids are exclusively selected under constrained costs, and electric vehicles are almost not selected under 10000 constraints. The trends appear the same, however, with it almost always being more optimal to invest in technology up-front.

\subsubsection{Annual Vehicle Kilometres Travelled}

A normal distribution with a mean of $21562 \mathrm{~km}$ and a standard deviation of $4000 \mathrm{~km}$ was used in this Monte Carlo analysis. This resulted in a maximum yearly driven distance of $35095 \mathrm{~km}$ and a minimum driven distance of $8861 \mathrm{~km}$. A discount rate of $0 \%$ was assumed to generate Fig. 12, and it shows that greater efficiency is optimal without constraints. In Fig. 13, it can be clearly seen that there is a linear increase in TCO, with an infection point where electric vehicles are selected beyond full hybrids. Also evident from the figure is that for vehicles driven less than $10000 \mathrm{~km}$ per year, it becomes optimal to select start-stop hybrids even in the unconstrained case due to the lack of driving time which is available to recoup the initial investment. This effect was verified by increasing the discount rate, and it was noticed that even in the unconstrained up-front cost case, the vehicle selected was the start-stop hybrid for low utilization.

\subsubsection{Cost of Fuel}

A normal distribution with a mean of $0.95 \$ / L$ and a standard deviation of 0.2 was used in this Monte Carlo analysis. This resulted in a maximum price of $1.60 \$ / \mathrm{L}$ and a minimum price of $0.22 \$ / \mathrm{L}$. The discount rate was set at $0 \%$, and up-front costs at $\$ 10000$ and $\$ 1000$ respectively for comparison. As in the driven $\mathrm{km}$ sensitivity case, it was noted that full hybrids were selected until a fuel price of just over $1.1 \$ / \mathrm{L}$, after which electric

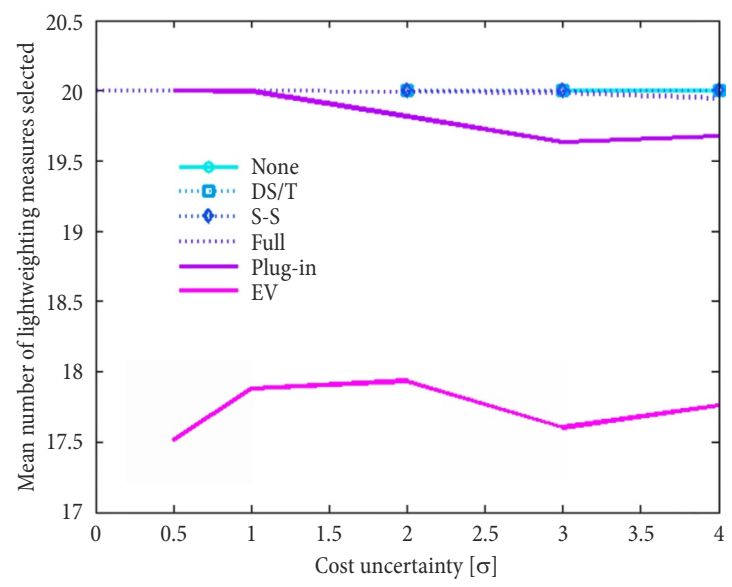

Fig. 11. As cost uncertainty increases, the number of lightweighting technologies selected decreases slightly 


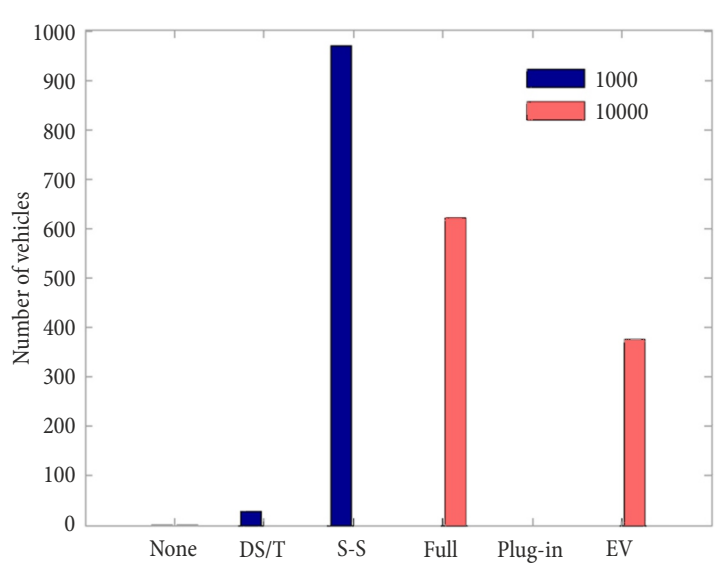

Fig. 12. At $0 \%$ discount rate, it is always optimal to select greater efficiency measures (legend refers to maximum up-front cost in \$)

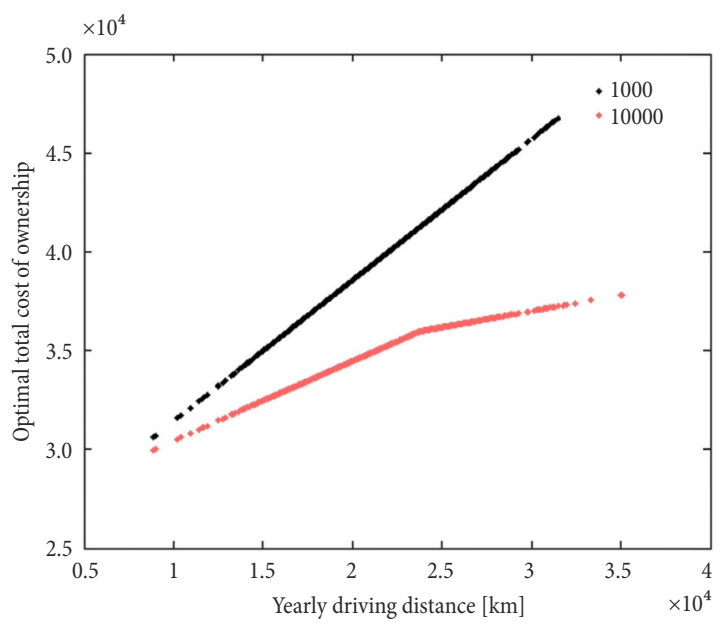

Fig. 13. After about $25000 \mathrm{~km}$ of driving, electric vehicles become cost-optimal, and sensitivity goes down (below $10000 \mathrm{~km}$ of driving, regardless of technology cost cap, start-stop hybrids become optimal)

vehicles were selected. When discount rate is increased to $5 \%$, at a fuel cost of below $0.3 \$ / \mathrm{L}$, non-hybrids, and start-stop hybrids begin to be selected regardless of the cap on up-front technology cost. At a fuel cost above \$1.4, EV's begin to be optimal, as shown in Fig. 14.

\subsubsection{Lifetime Parameters}

A normal distribution with a mean of 11 years and a standard deviation of 3 was used in this Monte Carlo analysis. This resulted in a maximum lifetime of 24 years and a minimum lifetime of 3 years. Only integer year lifetimes were considered. The discount rate was set at $0 \%$, and the maximum up-front cost at $\$ 10000$ and $\$ 1000$. As in the previous two cases, Fig. 15 shows that after about 12 years of ownership, electric vehicles are selected before full hybrids, and sensitivity decreases. As previously found, it is always optimal to select non-hybrids for vehicles owned for fewer than around 4 years, regardless of the cap on technology cost and even at a $0 \%$ discount rate, suggesting that these decisions are truly sensitive to the length of vehicle ownership.

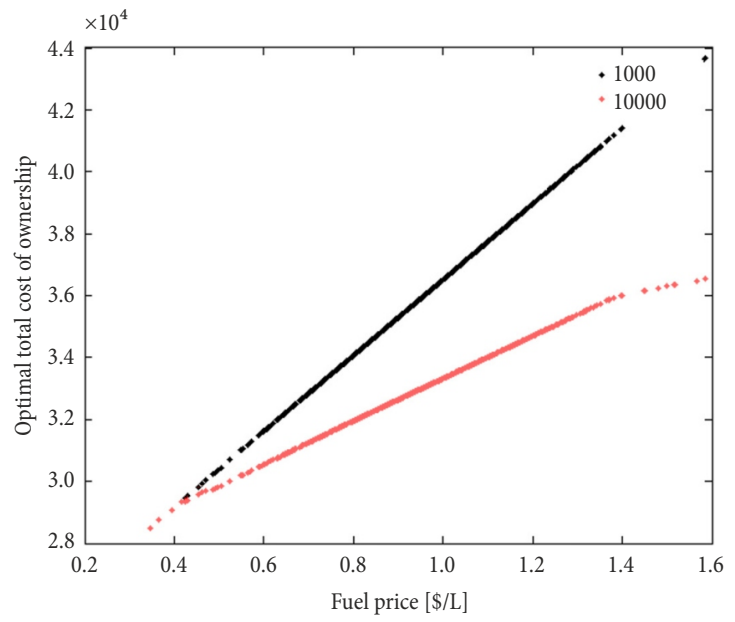

Fig. 14. Fuel price below 1.4 \$/L result in full hybrids being selected for unconstrained cases, and prices below $0.3 \$ / \mathrm{L}$ result in non-hybrids start-stop hybrids being selected for all up-front cost cases at 5\% discount rate

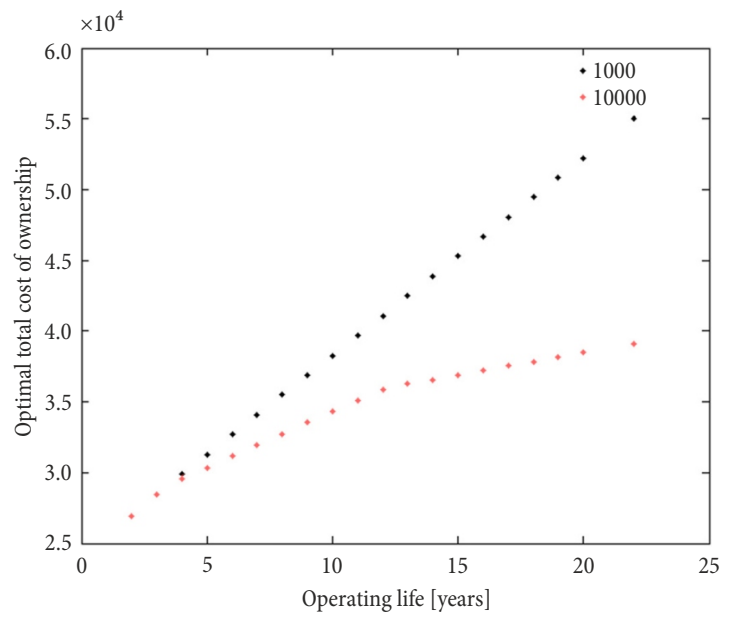

Fig. 15. Ownership below 4 years results in non-hybrids being optimal for all up-front cost constraint cases even at $0 \%$ discount rate

\subsubsection{Discount Rate}

The sensitivity of the results to discount rate is shown in Fig. 16. It is clear that, as expected, the greater the degree of future cost discounting, the lower the overall TCO appears, since future costs are less important. This translates into the exclusive selection of the technology with the lowest up-front cost, namely the non-hybridized case. Interestingly, even at a 50\% discount rate, many of the lightweighting technologies with non-negative costs are still selected (info, gage, and warning system, electrical dis. and electronic control system, exhaust system, fuel system, engine system, suspension system), a factor of the high weight reductions with low marginal costs of these technologies. As the maximum tolerable up-front cost increases, the higher the discount rate, the more stable the TCO remains, as is expected since in general fewer technologies are chosen. High discount rates lead to the selection of the cheapest up-front technologies. 
a)

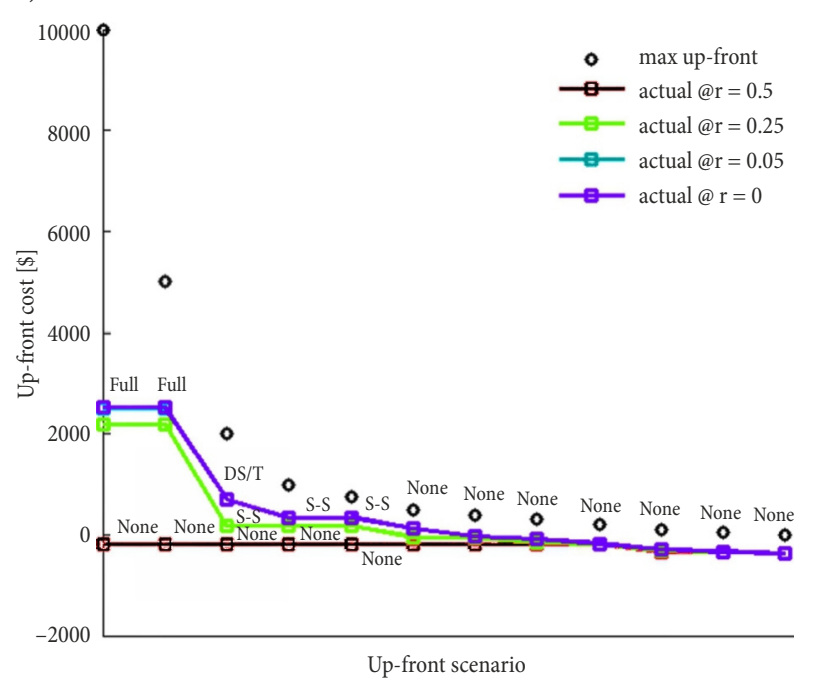

b)

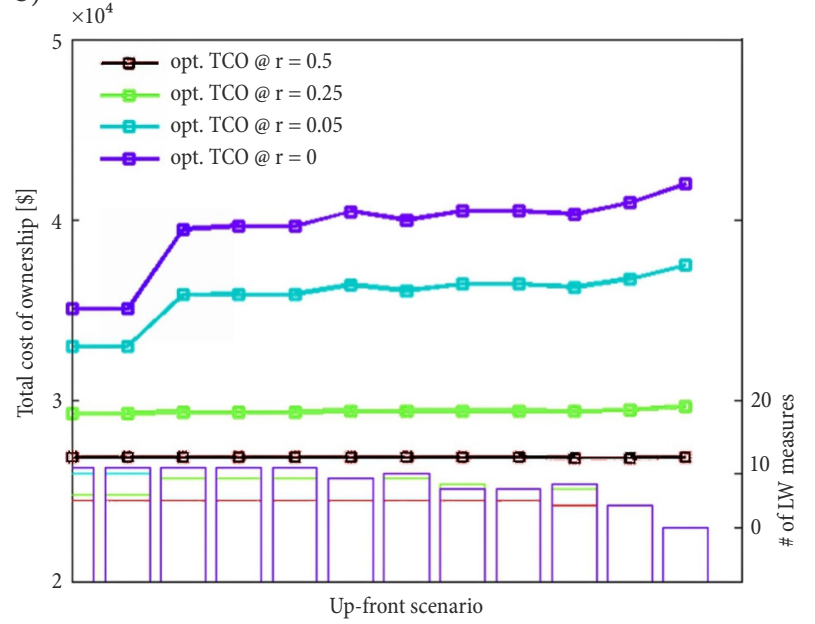

Fig. 16. As the discount rate increases, the cheaper up-front technologies are preferred (many lightweighting technologies with positive up-front costs were still selected at high discount rates)

\section{Conclusions}

In this work, a set of technology options were proposed to study the decision-making methods, which could be applied to optimally choose degrees of lightweighting and efficiency technologies. It was found that:

- the fundamental relationships between energy use, mass, and efficiency were invoked to derive a simple explanation for the reduction in the marginal effectiveness of weight reduction under changing efficiencies;

- by applying first-principles and a dynamic programming algorithm, an optimal set of efficiency and lightweighting options can be determined, and at $0 \%$ discount rate with a cap of $\$ 1000$ to spend up-front, all of the lightweighting technologies are selected;

- uncertainty in future costs for lightweighting technologies result in overall fewer lightweighting technologies being selected, in a manner consistent with the findings of the paper (Wilhelm et al. 2012), but for reasons which are now more clearly illuminated relating to the probability of establishing a negative value through a higher up-front cost;

- uncertainty in future costs of efficiency technologies result in a broader set of technologies being considered optimal, and a global increase in the mean total cost of ownership;

- uncertainty in both technologies results in the likely TCO rising regardless of the distribution chosen, even which cost decrease is more probable; this is relevant, as it suggests that even if technology costs decrease more often than they increase, there is still enough positive price pressure from the uncertainty to push to lower adoption of advanced technology;

- contrary to the paper (Wilhelm et al. 2012), this work finds that despite increasing uncertainty, there is still an optimal mix of lightweighting and efficiency technology application; this discrepancy arises from the more rigorous treatment of uncertainty in this paper, and results in a more refined picture of the optimization problem;

- the optimal mix of lightweighting and efficiency technology is most sensitive to up-front cost or commensurately the discount rate;

- paying a higher up-front cost is always advantageous relative to lower up-front costs, at assumed $0 \%$ discount rate; as the discount rate rises, the point at which up-front costs justify efficiency investment for various technologies shifts to lower yearly costs;

- as operation costs sink, and discount rate increases, non-hybrids start-stop hybrids are considered more often as optimal; tipping points were identified for various fuel cost, operation length, and utilization rates;

- the optimal mix of efficiency technologies and lightweighting technologies is highly sensitive to discount rate; the higher the discount rate, the less expensive the efficiency package selected, and the fewer lightweighting technologies are selected; even at a 50\% discount rate, however, 8 lightweighting technologies are still optimal for non-hybrids.

Future work should develop a multi-objective optimization to study the impact, which corporate average fuel economy standards have in the lightweighting/efficiency technology optimization framework developed here. As well, other criteria such as space, volume, safety feature impingements could be considered more rigorously. Additional vehicle platforms should be studied to validate the generalizability of these methods. As well, the impact that reduced maintenance costs achievable via powertrain electrification should be added to the TCO calculation.

\section{Acknowledgements}

The Authors would like to acknowledge the support of the SUTD-MIT International Design Centre (Singapore) in enabling this research. 


\section{References}

Allcott, H.; Wozny, N., 2012. Gasoline prices, fuel economy, and the energy paradox, The Review of Economics and Statistics 96(5): 779-795. https://doi.org/10.1162/REST_a_00419

Cheah, L.; Evans, C.; Bandivadekar, A.; Heywood, J. 2009. Factor of two: halving the fuel consumption of new U.S. automobiles by 2035, in J. S. Cannon, D. Sperling (Eds.). Reducing Climate Impacts in the Transportation Sector, 49-71. https://doi.org/10.1007/978-1-4020-6979-6_4

FEV. 2012. Light-Duty Vehicle Mass Reduction and Cost Analysis: Midsize Crossover Utility Vehicle. Report No. EPA-420-R-12-026. US Environmental Protection Agency (EPA). 878 p.

Greene, D. 2010b. Why the New Market for New Passenger Cars Generally Undervalues Fuel Economy. OECD/ITF Joint Transport Research Centre Discussion Papers. Discussion Paper No. 2010-6.

https://doi.org/10.1787/5kmjp68qtm6f-en

Greene, D. L. 2010a. How Consumers Value Fuel Economy: a Literature Review. Report No. EPA-420-R-10-008. US Environmental Protection Agency (EPA). 79 p.

Guzzella, L.; Sciarretta, A. 2015. Vehicle Propulsion Systems: Introduction to Modeling and Optimization. 3rd edition. Springer. $412 \mathrm{p}$.

Klier, T.; Linn, J. 2012. New-vehicle characteristics and the cost of the corporate average fuel economy standard, The RAND Journal of Economics 43(1): 186-213.

https://doi.org/10.1111/j.1756-2171.2012.00162.x

Lieberman, J. 2009. LA 2009: Volkswagen Up! Lite Concept Teases with Practical, Lightweight Innovation. Available from Internet: http://www.autoblog.com/2009/12/02/volkswagen-up-lite-concept-at-2009-la-auto-show

Magee, C. 1982. The role of weight reducing materials in automotive fuel savings, SAE Technical Paper 820147. https://doi.org/10.4271/820147

Martello, S.; Toth, P. 1990. Knapsack Problems: Algorithms and Computer Implementations. Wiley. 308 p.

MathWorks. 2014. Triangular Distribution. MATLAB \& Simulink. Available from Internet: https://se.mathworks.com/ help/stats/triangular-distribution.html
Pagerit, S.; Sharer, P.; Rousseau, A. 2006. Fuel economy sensitivity to vehicle mass for advanced vehicle powertrains, SAE Technical Paper 2006-01-0665.

https://doi.org/10.4271/2006-01-0665

Petter. 2009. File Exchange: 0-1 Knapsack. MATLAB Central. Available from Internet: https://se.mathworks.com/matlabcentral/fileexchange/22783-0-1-knapsack

Ramsbrock, J.; Vilimek, R.; Weber, J. 2013. Exploring electric driving pleasure - the BMW EV pilot projects, Lecture Notes in Computer Science 8005: 621-630. https://doi.org/10.1007/978-3-642-39262-7_70

EIA. 2014. Electricity. Data. US Energy Information Administration (EIA). Available from Internet: http://www.eia.gov/ electricity/data.cfm

EIA. 2012. Total Energy: Annual Energy Review. Table 5.24: Retail Motor Gasoline and On-Highway Diesel Fuel Prices, 1949-2011 (Dollars per Gallon). US Energy Information Administration (EIA). Available from Internet: http://www. eia.gov/totalenergy/data/annual/showtext.php?t=ptb0524

Verbrugge, M.; Lee, T.; Krajewski, P. E.; Sachdev, A. K.; Bjelkengren, C.; Roth, R.; Kirchain, R. 2009. Mass decompounding and vehicle lightweighting, Materials Science Forum 618-619: 411-418.

https://doi.org/10.4028/www.scientific.net/MSF.618-619.411

Wilhelm, E.; Hofer, J.; Schenler, W.; Guzzella, L. 2012. Optimal implementation of lightweighting and powertrain efficiency technology in passengers' vehicles, Transport 27(3): 237-249. https://doi.org/10.3846/16484142.2012.719546

Wilhelm, E. J.; Schenler, W. W. 2010. Reducing the weight of electric, hybrid, and fuel-cell vehicles using heuristic design, in Proceedings of the 2010 International Advanced Mobility Forum, 9-10 March 2010, Geneva, Switzerland. (USB).

Wohlecker, R.; Johannaber, M.; Espig, M. 2007. Determination of weight elasticity of fuel economy for ICE, hybrid and fuel cell vehicles, SAE Technical Paper 2007-01-0343. https://doi.org/10.4271/2007-01-0343

\section{APPENDIX A}

\begin{tabular}{|l|c|c|c|c|c|}
\hline \multicolumn{1}{|c|}{ System } & $\begin{array}{c}\text { System } \\
\text { mass } \\
\text { reduction } \\
{[\mathrm{kg}]}\end{array}$ & $\begin{array}{c}\text { System } \\
\text { incremental direct } \\
\text { manufacturing } \\
\text { cost impact }[\$]\end{array}$ & $\begin{array}{c}\text { System } \\
\text { incremental } \\
\text { tooling impact } \\
\text { cost [\$ } \times 1000]\end{array}$ & $\begin{array}{c}\text { Average system } \\
\text { cost/kilogram } \\
\text { with tooling } \\
{[\$ / \mathrm{kg}]}\end{array}$ & $\begin{array}{c}\% \text { vehicle } \\
\text { mass } \\
\text { reduction }\end{array}$ \\
\hline Transmission system & 18.90 & $(114.15)$ & $(7650.80)$ & $(6.26)$ & 1.10 \\
\hline Body system (Group A) BIW an closures & 68.32 & $(227.45)$ & $(22900.00)$ & $(3.51)$ & 3.99 \\
\hline $\begin{array}{l}\text { Body system (Group D) glazing and body } \\
\text { mechatronics }\end{array}$ & 6.16 & $(15.25)$ & 0.00 & $(2.48)$ & 0.36 \\
\hline Lighting system & 0.53 & $(0.76)$ & 400.00 & $(1.01)$ & 0.03 \\
\hline Driveline system & 1.50 & $(0.16)$ & $(685.86)$ & $(0.36)$ & 0.09 \\
\hline Frame and mounting system & 16.34 & $(3.28)$ & $(3700.39)$ & $(0.32)$ & 0.95 \\
\hline Fluid and misc & 0.00 & 0.00 & 0.00 & 0.00 & 0.00 \\
\hline Exhaust system & 7.52 & 2.47 & 0.00 & 0.33 & 0.44 \\
\hline Fuel system & 12.70 & 3.91 & 1625.30 & 0.38 & 0.74 \\
\hline Engine system & 30.25 & 33.69 & 5892.20 & 1.22 & 1.77 \\
\hline Electrical dis. and electronic control system & 0.89 & 1.35 & 103.50 & & 1.58 \\
\hline Suspension system & 66.83 & 144.71 & $(7544.37)$ & 2.10 & 3.91 \\
\hline
\end{tabular}


End of Appendix A

\begin{tabular}{|l|c|c|c|c|c|}
\hline \multicolumn{1}{|c|}{ System } & $\begin{array}{c}\text { System } \\
\text { mass } \\
\text { reduction } \\
{[\mathrm{kg}]}\end{array}$ & $\begin{array}{c}\text { System } \\
\text { incremental direct } \\
\text { manufacturing } \\
\text { cost impact }[\$]\end{array}$ & $\begin{array}{c}\text { System } \\
\text { incremental } \\
\text { tooling impact } \\
\text { cost }[\$ \times 1000]\end{array}$ & $\begin{array}{c}\text { Average system } \\
\text { cost/kilogram } \\
\text { with tooling } \\
{[\$ / \mathrm{kg}]}\end{array}$ & $\begin{array}{c}\% \text { vehicle } \\
\text { mass } \\
\text { reduction }\end{array}$ \\
\hline Info, gage, and warning system & 0.08 & 0.19 & 0.00 & 2.45 & 0.00 \\
\hline In-vehicle entertainment system & 1.07 & 2.35 & 1175.60 & 2.79 & 0.06 \\
\hline Body system (Group B) interior & 42.00 & 122.98 & 9966.15 & 3.06 & 2.45 \\
\hline Body system (Group C) exterior & 2.37 & 7.52 & 0.00 & 3.17 & 0.14 \\
\hline Climate control system & 2.44 & 9.34 & 386.00 & 3.92 & 0.14 \\
\hline Brake system & 32.75 & 169.56 & $(1426.12)$ & 5.15 & 1.91 \\
\hline Steering system & 1.82 & 11.05 & 1352.70 & 6.48 & 0.11 \\
\hline Vehicle & 312.47 & 148.07 & $(23006.09)$ & 0.43 & 18.24 \\
\hline
\end{tabular}

\section{APPENDIX B}

\begin{tabular}{|c|c|c|c|c|c|}
\hline No & Model & Powertrain & $\begin{array}{c}\text { Weight } \\
{[\mathrm{kg}]}\end{array}$ & $\begin{array}{c}\text { MSRP } \\
{[\$]}\end{array}$ & $\begin{array}{c}\text { Fuel } \\
\text { consumption } \\
{[\mathrm{L} / 100 \mathrm{~km}]}\end{array}$ \\
\hline 1 & 2014 Fusion FWD - Baseline & 2.5 L 4 cylinders, automatic & 1643 & 23935 & 9.0 \\
\hline 2 & 2014 Fusion FWD & 2.0 L 4 cylinders, automatic, turbo & 1603 & 25591 & 9.0 \\
\hline 3 & 2014 Fusion FWD - Downsize/turbo & $1.5 \mathrm{~L} 4$ cylinders, turbo & 1643 & 24730 & 8.4 \\
\hline 4 & 2014 Fusion FWD - Start-stop & $1.5 \mathrm{~L} 4$ cylinders, turbo w start-stop & 1643 & 24230 & 8.1 \\
\hline 5 & 2014 Chevrolet Malibu & 2.5 L, 4 cylinders, automatic (S6) & 1664 & 22140 & 8.1 \\
\hline 6 & 2014 Chevrolet Malibu & 2.0 L, 4 cylinders, automatic (S6), Turbo & 1655 & 26750 & 9.8 \\
\hline 7 & 2014 Chevrolet Malibu eAssist & 2.4 L, 4 cylinders, automatic (S6) & 1633 & 25845 & 8.1 \\
\hline 8 & 2014 Toyota Prius & Split parallel-series & 1383 & 30005 & 4.7 \\
\hline 9 & 2014 Honda Accord Hybrid & Transmission-less & 1614 & 29155 & 5.0 \\
\hline 10 & 2014 Ford Fusion Hybrid FWD - Full hybrid & Split parallel-series & 1643 & 27280 & 5.0 \\
\hline 11 & 2014 Ford C-max Hybrid FWD & Split parallel-series & 1640 & 25170 & 5.5 \\
\hline 12 & 2014 Honda Insight & Integrated Motor Assist (IMA) & 1249 & 18725 & 5.5 \\
\hline 13 & 2014 Toyota Camry Hybrid LE & Split parallel-series & 1602 & 27670 & 5.7 \\
\hline 14 & 2013 Hyundai Sonata Hybrid & Transmission-mounted (IMA) & 1571 & 25650 & 6.2 \\
\hline 15 & 2013 Kia Optima Hybrid & Transmission-mounted (IMA) & 1589 & 25900 & 6.2 \\
\hline 16 & 2014 Toyota Prius Plug-in Hybrid & Plug-in: split parallel-series & 1439 & 34905 & 4.7 \\
\hline 17 & 2014 Honda Accord Plug-in Hybrid & Plug-in: transmission-less & 1727 & 39780 & 5.1 \\
\hline 18 & $\begin{array}{l}2014 \text { Ford C-max Energi Plug-in Hybrid - } \\
\text { Plug-in Hybrid }\end{array}$ & Plug-in: split parallel-series & 1754 & 32920 & 3.6 \\
\hline 19 & 2014 Ford Fusion Energi Plug-in Hybrid & Plug-in: split parallel-series & 1779 & 36500 & 5.5 \\
\hline 20 & 2014 Chevrolet Volt & Plug-in: series hybrid & 1721 & 34185 & 6.4 \\
\hline 21 & 2014 Ford Focus Electric - Electric drive & Electric drive & 1647 & 35170 & 2.2 \\
\hline 22 & 2014 Nissan Leaf & Electric drive & 1474 & 28980 & 2.1 \\
\hline 23 & 2014 Chevrolet Spark EV & Electric drive & 1359 & 26685 & 2.0 \\
\hline 24 & 2014 Smart Fortwo electric drive coupe & Electric drive & 1152 & 25000 & 2.2 \\
\hline
\end{tabular}

